Yamada, T.

Osaka J. Math.

39 (2002), 363-381

\title{
HARMONIC COHOMOLOGY GROUPS ON COMPACT SYMPLECTIC NILMANIFOLDS
}

\author{
TAKUMI YAMADA
}

(Received January 28, 2000)

\section{Introduction}

Let $\left(M^{2 m}, \omega\right)$ be a symplectic manifold. Brylinski [2] defined the star operator $*: \Omega^{k}(M) \rightarrow \Omega^{2 m-k}(M)$ for the symplectic structure $\omega$ as an analogy of the star operator for an oriented Riemannian manifold, where $\Omega^{k}(M)$ denotes the space of all $k$-forms on $M$, and also defined an operator $d^{*}=(-1)^{k} * d *: \Omega^{k}(M) \rightarrow \Omega^{k-1}(M)$. Now a form $\alpha$ on $M$ is called a symplectic harmonic form if it satisfies $d \alpha=d^{*} \alpha=0$. We denote by $\mathcal{H}^{k}(M)$ the space of all harmonic $k$-forms on $M$. We define symplectic harmonic $k$-cohomology group $H_{h r}^{k}(M)$ by $\mathcal{H}^{k}(M) /\left(B^{k}(M) \cap \mathcal{H}^{k}(M)\right)$. Brylinski conjectured that any de Rham cohomology class contains a harmonic representation. However, Mathieu [6] proved the following result:

Mathieu's Theorem. Let $\left(M^{2 m}, \omega\right)$ be a symplectic manifold of dimension $2 m$. Then following two assertions are equivalent:

(a) For any $k$, the cup-product $[\omega]^{k}: H_{D R}^{m-k}(M) \rightarrow H_{D R}^{m+k}(M)$ is surjective.

(b) For any $k, H_{D R}^{k}(M)=H_{h r}^{k}(M)$.

In particular, we see that if $M$ is a compact Kähler manifold, then any de Rham cohomology class contains a symplectic harmonic cocycle. Yan [11] gave a simpler, more direct proof of Mathieu's Theorem. Mathieu [6] also proved that, for $k=0,1,2$, $H_{D R}^{k}(M)=H_{h r}^{k}(M)$.

In this paper we study compact symplectic nilmanifolds. Let $\mathfrak{g}$ be a Lie algebra and put $\mathfrak{g}^{(0)}=\mathfrak{g}$ and let $\mathfrak{g}^{(i+1)}=\left[\mathfrak{g}, \mathfrak{g}^{(i)}\right]$ for $i \geq 0$. We say that a Lie algebra $\mathfrak{g}$ is $(r+1)$-step nilpotent if $\mathfrak{g}^{(r)} \neq(0)$ and $\mathfrak{g}^{(r+1)}=(0)$. A Lie group $G$ is called $(r+1)$-step nilpotent if its Lie algebra $\mathfrak{g}$ is $(r+1)$-step nilpotent. If $G$ is a simplyconnected $(r+1)$-step nilpotent Lie group and $\Gamma$ is a lattice of $G$, that is, a discrete subgroup of $G$ such that $G / \Gamma$ is compact, then we say that $G / \Gamma$ is a compact $(r+1)$-step nilmanifold. We also identify $\wedge \mathfrak{g}^{*}$ with the space of all left $G$-invariant forms on $G$. Nomizu [8] proved that, for each $k$, the Lie algebra cohomology group $H^{k}(\mathfrak{g})=Z^{k}(\mathfrak{g}) / B^{k}(\mathfrak{g})=\left(\operatorname{Ker} d \cap \bigwedge^{k}\left(\mathfrak{g}^{*}\right)\right) /\left(\operatorname{Im} d \cap \bigwedge^{k}\left(\mathfrak{g}^{*}\right)\right)$ is isomorphic to the de Rham cohomology group $H_{D R}^{k}(M)=Z^{k}(M) / B^{k}(M)=\left(\operatorname{Ker} d \cap \Omega^{k}(M)\right) /\left(\operatorname{Im} d \cap \Omega^{k}(M)\right)$, where $M=G / \Gamma$. 
Benson and Gordon [1] have proved that the Hard Lefschetz Theorem fails for any symplectic structure on a non-toral compact nilmanifold to show that a non-toral compact nilmanifold does not admit any Kähler structure. The proof of Benson and Gordon also implies that the dimension of $H_{h r}^{2 m-1}(M)$ is not equal to the dimension $H_{h r}^{1}(M)$ for a non-toral compact nilmanifold.

For a left $G$-invariant symplectic form $\omega$ on a compact nilmanifold $G / \Gamma$, we denote by $\mathcal{H}^{k}(\mathfrak{g})$ the space of all left $G$-invariant harmonic forms on $G / \Gamma$. Moreover we define a subspace of Lie algebra cohomology group $H^{k}(\mathfrak{g})$ by $H_{h r}^{k}(\mathfrak{g})=\mathcal{H}^{k}(\mathfrak{g}) /\left(B^{k}(\mathfrak{g}) \cap\right.$ $\left.\mathcal{H}^{k}(\mathfrak{g})\right)$.

Let $M$ be a compact manifold and $\omega, \omega^{\prime}$ symplectic forms on $M$. We denote $\omega$-harmonic ( $\omega^{\prime}$-harmonic) $q$-cohomology group by $H_{\omega-h r}^{q}(M)\left(H_{\omega^{\prime}-h r}^{q}(M)\right)$. If for some $k$, the dimension of $H_{\omega-h r}^{k}(M)$ and $H_{\omega^{\prime}-h r}^{k}(M)$ are not equal, then there exists no diffeomorphisms $\varphi: M \longrightarrow M$ such that $\varphi^{*} \omega=\omega^{\prime}$. Thus harmonic cohomology groups play an important role in the classification of symplectic forms.

We are also interested in the following question raised by B. Khesin and D. McDuff (see Yan [11]).

Question : On which compact manifold $M$, does there exist a family $\omega_{t}$ of symplectic forms such that the dimension of $H_{h r}^{k}(M)$ varies?

This question was considered by Yan [11] for compact symplectic 4-manifolds and he constructed compact 4-manifold which have a family $\omega_{t}$ of symplectic forms such that the dimension of $H_{\omega_{t}-h r}^{3}(M)$ varies. Yan also observed that for compact 4-dimensional nilmanifolds the dimension $H_{\omega_{t}-h r}^{3}(M)$ is independent of symplectic forms. Now we consider the following question.

Question : On which compact nilmanifold $M$, does there exist a family $\omega_{t}$ of symplectic forms such that the dimension of $H_{h r}^{k}(M)$ varies?

In Section 4, we prove

Proposition 1. Let $M^{2 m}$ be a compact manifold and $\omega, \omega^{\prime}$ symplectic forms on $M$ such that $\omega-\omega^{\prime}=d \gamma$ for some $\gamma \in \Omega^{1}(M)$ (it is not necessary that $M$ is a nilmanifold). Then we have, for each $q$,

$$
H_{\omega-h r}^{q}(M)=H_{\omega^{\prime}-h r}^{q}(M) .
$$

Proposition 2. Let $(M=G / \Gamma, \omega)$ be a compact symplectic nilmanifold. Then we have

$$
H_{\omega-h r}^{q}(M)=H_{\omega_{0}-h r}^{q}(M)=H_{\omega_{0}-h r}^{q}(\mathfrak{g}),
$$

where $\omega_{0}$ is a left $G$-invariant closed 2-form such that $\omega-\omega_{0}=d \gamma$ for some $\gamma \in$ $\Omega^{1}(M)$.

In Section 5, we prove the following: 
Theorem 3. Let $M^{2 m}=G / \Gamma$ be a compact $(r+1)$-step nilmanifold. Then for any symplectic structure $\omega$ on $M$, we have

$$
\operatorname{dim} H_{h r}^{1}(M)-\operatorname{dim} H_{h r}^{2 m-1}(M) \geq \operatorname{dim} \mathfrak{g}^{(r)} .
$$

In particular, if $M$ is a 2-step nilmanifold, then

$$
\operatorname{dim} H_{h r}^{1}(M)-\operatorname{dim} H_{h r}^{2 m-1}(M)=\operatorname{dim}[\mathfrak{g}, \mathfrak{g}] .
$$

Let $G$ be a simply-connected nilpotent Lie group and $\mathfrak{g}$ be its Lie algebra. Note that $G$ has a lattice if and only if $\mathfrak{g}$ admits a basis with respect to which the constants of structure are rational (see Raghunathan [9] Theorem 2.12 of Chapter II).

In Section 7, we prove the following:

Theorem 4. Let $\mathfrak{g}$ be the 2-step nilpotent Lie algebra for dimension 6 of the form

$$
\mathfrak{g}=\operatorname{span}\left\{X_{1}, X_{2}, X_{3}, X_{4}, X_{5}, X_{6}\right\}
$$

where

$$
\left[X_{1}, X_{6}\right]=X_{5},\left[X_{1}, X_{4}\right]=X_{3},\left[X_{4}, X_{6}\right]=X_{2}
$$

and $\omega_{1}, \omega_{2}, \omega_{3}, \omega_{4}, \omega_{5}, \omega_{6}$ denote its dual basis. Moreover, let

$$
\omega=\omega_{1} \wedge \omega_{3}+\omega_{2} \wedge \omega_{4}+\omega_{5} \wedge \omega_{6}
$$

and

$$
\omega^{\prime}=-2 \omega_{1} \wedge \omega_{2}-\omega_{3} \wedge \omega_{6}-\omega_{4} \wedge \omega_{5}
$$

Then $\left\{\omega_{t}=(1-t) \omega+t \omega^{\prime} ; \mathbb{R} \ni t \neq x_{0}\right\}$ is a family of symplectic forms on compact nilmanifold $G / \Gamma$, where $x_{0}\left(x_{0} \fallingdotseq-3.8473\right)$ is a unique real solution for $1-3 t+3 t^{2}+$ $t^{3}=0$, such that

$$
\begin{array}{ll}
\text { for } \omega_{0}=\omega, & \operatorname{dim} H_{h r}^{2}(G / \Gamma)-\operatorname{dim} H_{h r}^{4}(G / \Gamma)=1 \\
\text { for } \omega_{t}\left(t \neq 0, x_{0}\right), & \operatorname{dim} H_{h r}^{2}(G / \Gamma)-\operatorname{dim} H_{h r}^{4}(G / \Gamma)=0 .
\end{array}
$$

Since $H_{D R}^{2}(G / \Gamma)=H_{\omega-h r}^{2}(G / \Gamma)$ for any symplectic forms, we have 6-dimensional nilmanifold which has a family $\omega_{t}$ of symplectic forms such that the dimension of $H_{\omega_{t}-h r}^{4}(M)$ varies.

The author would like to express his deep appreciation to Professor Yusuke Sakane for his thoughtful guidance and encouragement given during the completion of this paper. 


\section{Operators on $\Omega^{*}(M)$ and the relations}

In this section, we define some operators on a symplectic manifold and study their relations.

Let $\left(M^{2 m}, \omega\right)$ be a symplectic manifold. We define a star operator $*_{\omega}=$ $*: \Omega^{k}(M) \rightarrow \Omega^{2 m-k}(M)$. Let $\mathbf{G}$ be the skew symmetric bivector field dual to $\omega$.

By the Darboux's theorem, we can write in canonical coordinates

$$
\omega=d p_{1} \wedge d q_{1}+\cdots+d p_{m} \wedge d q_{m}
$$

and

$$
\mathbf{G}=\frac{\partial}{\partial q_{1}} \wedge \frac{\partial}{\partial p_{1}}+\cdots+\frac{\partial}{\partial q_{m}} \wedge \frac{\partial}{\partial p_{m}} .
$$

We define a star operator

$$
*: \Omega^{k}(M) \rightarrow \Omega^{2 m-k}(M) \quad \text { for } \quad k=0, \ldots, 2 m
$$

by

$$
\alpha \wedge * \beta=\left(\wedge^{k}(\mathbf{G})\right)(\alpha, \beta) v_{M} \text { for } \alpha, \beta \in \Omega^{k}(M),
$$

where $v_{M}=\omega^{m} / m !$.

We also define an operator $d^{*}$ by $d^{*}=(-1)^{k} * d *: \Omega^{k}(M) \rightarrow \Omega^{k-1}(M)$.

Definition 2.1. For a symplectic manifold $(M, \omega)$, a $k$-form $\alpha \in \Omega^{k}(M)$ is called $\omega$-harmonic or simply, harmonic, if it satisfies

$$
d^{*} \alpha=d \alpha=0
$$

We denote by $\mathcal{H}_{\omega}^{k}(M)=\mathcal{H}^{k}(M)$ the space of all harmonic $k$-forms. We define symplectic harmonic $k$-cohomology group $H_{\omega-h r}^{k}(M)=H_{h r}^{k}(M)=\mathcal{H}^{k}(M) /\left(B^{k}(M) \cap\right.$ $\left.\mathcal{H}^{k}(M)\right)$. We also define $L_{\omega}=L: \Omega^{k}(M) \rightarrow \Omega^{k+2}(M)$ by $L(\alpha)=\alpha \wedge \omega$.

\section{Lemma 2.2.}

$$
d^{*}=[d, i(\mathbf{G})]
$$

Proof. See [2].

REMARK. Since $d^{* 2}=0$, we can define homology groups as follows.

$$
H_{k}^{h r}(M):=\mathcal{H}^{k}(M) /\left(B_{k}(M) \cap \mathcal{H}^{k}(M)\right),
$$


where $B_{k}(M)=\operatorname{Im} d^{*} \cap \Omega^{k}(M)$. For any $k$,

$$
*: H_{m-k}^{h r}(M) \rightarrow H_{h r}^{m+k}(M)
$$

is an isomorphism. In fact, for $\alpha \in \mathcal{H}^{m-k}(M)$, we have

$$
\begin{gathered}
d^{*}(* \alpha)=(-1)^{m+k} * d * * \alpha=(-1)^{m+k} * d \alpha=0 \\
d(* \alpha)=* * d * \alpha=(-1)^{m-k} * d^{*} \alpha=0 .
\end{gathered}
$$

Thus $* \alpha \in \mathcal{H}^{m+k}(M)$. Similarly, $*: B^{m-k}(M) \rightarrow B_{m+k}(M)$. Thus $*$-operator induces a homomorphism

$$
*: H_{m-k}^{h r}(M) \rightarrow H_{h r}^{m+k}(M) .
$$

We can also define $*: H_{h r}^{m+k} \rightarrow H_{m-k}^{h r}(M)$. Since $* *=I d$, we see that

$*: H_{m-k}^{h r}(M) \rightarrow H_{h r}^{m+k}(M)$ is an isomorphism (cf. [2]).

Now, we define operator $L^{*}$ by

$$
L^{*}=* L *: \Omega^{k}(M) \rightarrow \Omega^{k-2}(M) .
$$

We can easily see that $L^{*}$ is the adjoint operator for $L$, where we define an inner product (, ) on $\Omega^{*}(M)$ by $(\alpha, \beta)=\int *(\alpha \wedge * \beta) v_{M}$ for $\alpha, \beta \in \Omega^{k}(M)$.

Proposition 2.3 (Yan [11]).

$$
i(\mathbf{G})=-L^{*}
$$

Moreover, we define

$$
A=\sum(m-k) \pi_{k}
$$

where $\pi_{k}: \Omega^{*}(M) \rightarrow \Omega^{k}(M)$ is the natural projection.

These operators satisfy the following relations:

Proposition 2.4 (Yan $[11]$ ). $\quad\left[L^{*}, L\right]=A \quad[A, L]=-2 L \quad\left[A, L^{*}\right]=2 L^{*}$

\section{Duality on harmonic forms}

First we introduce the following definition. Let $X, H, Y$ be the standard basis of $\mathfrak{s l}(2, \mathbb{C})=\mathfrak{s l}(2)$ i.e.

$$
H=\left(\begin{array}{cc}
1 & 0 \\
0 & -1
\end{array}\right) \quad X=\left(\begin{array}{ll}
0 & 1 \\
0 & 0
\end{array}\right) \quad Y=\left(\begin{array}{ll}
0 & 0 \\
1 & 0
\end{array}\right) .
$$


Definttion 3.1. Let $V$ be the (infinite-dimensional) vector space of a Lie algebra representation. We say that $V$ is an $\mathfrak{s l}(2)$-module of finite $H$-spectrum if the following two conditions are satisfied:

(a) $V$ can be decomposed as the direct sum of eigenspace of $H$.

(b) $H$ has only finitely many distinct eigenvalues.

By a basic result on an $\mathfrak{s l}(2)-$-representation we have the following:

Proposition 3.2. Let $V$ be an $\mathfrak{s l}(2)$-module of finite $H$-spectrum. Then we have:

For any $k$, the maps

$$
Y^{k}: V_{k} \rightarrow V_{-k}
$$

and

$$
X^{k}: V_{-k} \rightarrow V_{k}
$$

are isomorphisms (where $V_{k}$ is an eigenspace of $H$ with eigenvalue $k$ ).

Now we can give a representation of $\mathfrak{s t}(2, \mathbb{R})$ on $\Omega^{*}(M)$ by the following correspondence:

$$
X \longleftrightarrow * L *, \quad Y \longleftrightarrow L, \quad H \longleftrightarrow A .
$$

We can easily see that $\Omega^{*}(M)$ is an $\mathfrak{s l}(2)$-module of finite $H$-spectrum. Thus we have

Proposition 3.3 (Duality on forms) (Yan [11]).

$$
L^{k}: \Omega^{m-k}(M) \rightarrow \Omega^{m+k}(M)
$$

is an isomorphism.

Moreover, since $\mathcal{H}^{*}(M)$ is an $\mathfrak{s l}(2, \mathbb{R})$-submodule of $\Omega^{*}(M)$, we have

Proposition 3.4 (Duality on harmonic forms) (Yan [11]).

$$
L^{k}: \mathcal{H}^{m-k}(M) \rightarrow \mathcal{H}^{m+k}(M)
$$

is an isomorphism.

For a left $G$-invariant symplectic form $\omega$ on a compact nilmanifold $G / \Gamma$, we denote by $\mathcal{H}^{k}(\mathfrak{g})$ the space of all left $G$-invariant harmonic forms on $G / \Gamma$.

Now we have the following: 
Proposition 3.5. Let $\left(M^{2 m}, \omega\right)$ be a compact symplectic nilmanifold such that $\omega \in \Lambda^{2}\left(\mathfrak{g}^{*}\right)$, then

$$
L^{k}: \mathcal{H}^{m-k}(\mathfrak{g}) \rightarrow \mathcal{H}^{m+k}(\mathfrak{g})
$$

is an isomorphism.

Proof. Let $\left\{X_{1}, \ldots, X_{2 m}\right\}$ be a basis of $\mathfrak{g}$ and $\left\{\omega_{1}, \ldots, \omega_{2 m}\right\}$ be its dual basis. Then $\omega$ can be written as

$$
\omega=\sum a_{i j} \omega_{i} \wedge \omega_{j} \quad a_{i j}=-a_{j i} \in \mathbb{R} .
$$

Further, it is easy to see that

$$
\mathbf{G}=-\sum c_{i j} X_{i} \wedge X_{j}
$$

where $\left(c_{i j}\right)$ is the inverse matrix for transpose matrix of $\left(a_{i j}\right)$. It follows that $\mathcal{H}^{*}(\mathfrak{g})$ is an $\mathfrak{s l}(2, \mathbb{R})$-submodule.

\section{Harmonic cohomology groups on $M$}

We need some lemmas to prove Theorem 1 .

Lemma 4.1 (Yan [11]). Let $(M, \omega)$ be a symplectic manifold. Then we have

$$
P_{\omega}^{m-k}(M) \subset H_{\omega-h r}^{m-k}(M)
$$

where

$$
P_{\omega}^{m-k}(M)=\left\{v \in H_{D R}^{m-k}(M) \mid L_{\omega}^{k+1} v=0\right\}
$$

Lemma 4.2. Let $M^{2 m}$ be a compact manifold and $\omega, \omega^{\prime}$ symplectic forms on $M$ such that $\omega-\omega^{\prime}=d \gamma$ for some $\gamma \in \Omega^{1}(M)$. Then we have

$$
P_{\omega}^{m-k}(M)=P_{\omega^{\prime}}^{m-k}(M)
$$

Proof. Let $v=[z] \in P_{\omega}^{m-k}(M)$, where $z \in Z^{m-k}(M)$. Since $\omega=\omega^{\prime}+d \gamma$, we have

$$
\begin{aligned}
L_{\omega}^{k+1} z & =\omega^{k+1} \wedge z \\
& =\left(\omega^{\prime}+d \gamma\right)^{k+1} \wedge z \\
& =L_{\omega^{\prime}}^{k+1} z+\sum_{r \neq k+1}\left(\begin{array}{c}
k+1 \\
r
\end{array}\right) \omega^{\prime} \wedge(d \gamma)^{k-r+1} \wedge z .
\end{aligned}
$$

Therefore,

$$
L_{\omega}^{k+1} v=\left[L_{\omega}^{k+1} z\right]=\left[L_{\omega^{\prime}}^{k+1} z\right]=L_{\omega^{\prime}}^{k+1} v=0
$$


which implies $v \in P_{\omega^{\prime}}^{m-k}(M)$, so that $P_{\omega}^{m-k}(M)=P_{\omega^{\prime}}^{m-k}(M)$.

Lemma 4.3. Let $(M, \omega)$ be a compact symplectic manifold. Then, for $k \geq 0$, we have

$$
H_{h r}^{m-k}(M)=P^{m-k}(M)+L\left(H_{h r}^{m-k-2}(M)\right)
$$

Proof. Let $a \in H_{h r}^{m-k}(M)$. Since $L^{k+2}: \mathcal{H}^{m-k-2}(M) \rightarrow \mathcal{H}^{m+k+2}(M)$ is an isomorphism, there exists $b=[\beta]$, where $\beta \in \mathcal{H}^{m-k-2}$ such that

$$
L^{k+1} a=L^{k+2} b .
$$

Thus

$$
L^{k+1}(a-b \wedge \omega)=0,
$$

which implies

$$
a-b \wedge \omega \in P^{m-k}(M) .
$$

Moreover, since $b \wedge \omega \in L\left(H_{h r}^{m-k-2}(M)\right)$, we get

$$
a=(a-b \wedge \omega)+b \wedge \omega \in P^{m-k}(M)+L\left(H_{h r}^{m-k-2}(M)\right) .
$$

Proposition 4.4. Let $M^{2 m}$ be a compact manifold and $\omega, \omega^{\prime}$ symplectic forms on $M$ such that $\omega-\omega^{\prime}=d \gamma$ for some $\gamma \in \Omega^{1}(M)$. Then we have, for each $q$,

$$
H_{\omega-h r}^{q}(M)=H_{\omega^{\prime}-h r}^{q}(M) .
$$

Proof. We prove our proposition by induction of the dimension of de Rham cohomology group. By the proof of Mathieu's theorem, we see $H_{D R}^{i}(M)=H_{h r}^{i}(M)$ for $i=0,1,2$ (cf. Corollary 8 of [6] and Corollary 3.1 of [11]). Therefore,

$$
H_{\omega-h r}^{i}(M)=H_{D R}^{i}(M)=H_{\omega^{\prime}-h r}^{i}(M) \text { for } i=0,1,2 .
$$

Assume that if $q<m-k$, then $H_{\omega-h r}^{q}(M)=H_{\omega^{\prime}-h r}^{q}(M)$. Let $[u] \in H_{\omega-h r}^{m-k-2}(M)$, where $u \in \mathcal{H}_{\omega}^{m-k-2}(M)$. By the assumption of induction, there exists $\left[u^{\prime}\right] \in H_{\omega^{\prime}-h r}^{m-k-2}(M)$, where $u^{\prime} \in \mathcal{H}_{\omega^{\prime}}^{m-k-2}(M)$ such that $[u]=\left[u^{\prime}\right] \in H_{D R}^{m-k-2}(M)$. Thus

$$
\begin{aligned}
L_{\omega}\left(H_{\omega-h r}^{m-k-2}(M)\right) \ni[\omega \wedge u] & =[\omega] \wedge[u] \\
& =[\omega] \wedge\left[u^{\prime}\right] \\
& =\left[\omega \wedge u^{\prime}\right]=\left[\left(\omega^{\prime}+d \gamma\right) \wedge u^{\prime}\right] \\
& =\left[\omega^{\prime} \wedge u^{\prime}\right] \in L_{\omega^{\prime}}\left(H_{\omega^{\prime}-h r}^{m-k-2}(M)\right) .
\end{aligned}
$$


Therefore, by Lemma 4.3

$$
\begin{aligned}
H_{\omega-h r}^{m-k}(M) & =P_{\omega}^{m-k}(M)+L_{\omega}\left(H_{\omega-h r}^{m-k-2}(M)\right) \\
& =P_{\omega^{\prime}}^{m-k}(M)+L_{\omega^{\prime}}\left(H_{\omega^{\prime}-h r}^{m-k-2}(M)\right) \\
& =H_{\omega^{\prime}-h r}^{m-k}(M) .
\end{aligned}
$$

Let $v=[z] \in H_{\omega-h r}^{m+k}(M)$, where $z \in \mathcal{H}_{\omega}^{m+k}(M)$. Since $L^{k}: \mathcal{H}^{m-k}(M) \rightarrow \mathcal{H}^{m+k}(M)$ is an isomorphism, there exists $w \in \mathcal{H}_{\omega}^{m-k}(M)$ such that $v=[z]=\left[L_{\omega}^{k} w\right]=L_{\omega}^{k}[w]$.

Thus by above argument, there exists $[u] \in H_{\omega^{\prime}-h r}^{m-k}(M)$, where $u \in \mathcal{H}_{\omega^{\prime}}^{m-k}(M)$ such that $[w]=[u]$. Since $\omega=\omega^{\prime}+d \gamma$, we have

$$
\begin{aligned}
L_{\omega}^{k}[w]=L_{\omega}^{k}[u] & =\left[\omega^{k} \wedge u\right] \\
& =\left[\left(\omega^{\prime}+d \gamma\right)^{k} \wedge u\right] \\
& =\left[L_{\omega^{\prime}}^{k} u+\sum_{r \neq k}\left(\begin{array}{l}
k \\
r
\end{array}\right) \omega^{\prime} \wedge(d \gamma)^{k-r} \wedge u\right]=\left[L_{\omega^{\prime}}^{k} u\right]=L_{\omega^{\prime}}^{k}[u],
\end{aligned}
$$

which implies

$$
H_{\omega-h r}^{q}(M)=H_{\omega^{\prime}-h r}^{q}(M) \quad(q=0, \ldots, 2 m) .
$$

For a left $G$-invariant symplectic form $\omega$ on $G / \Gamma$, let $H_{h r}^{k}(\mathfrak{g})=\mathcal{H}^{k}(\mathfrak{g}) /\left(B^{k}(\mathfrak{g}) \cap\right.$ $\left.\mathcal{H}^{k}(\mathfrak{g})\right)$ be a subspace of Lie algebra cohomology group $H^{k}(\mathfrak{g})$.

Proposition 4.5. Let $(M, \omega)$ be a symplectic nilmanifold such that $\omega$ is a left $G$-invariant closed 2-form. Then we have

$$
H_{h r}^{q}(M)=H_{h r}^{q}(\mathfrak{g}) \quad(q=0, \ldots, 2 m) .
$$

Proof. We prove our proposition by induction. Note that, since $d^{*}=[d, i(\mathbf{G})]$, $Z^{i}(\mathfrak{g})=\mathcal{H}^{i}(\mathfrak{g})$ for $i=0,1,2$. Applying Nomizu's theorem, for $i=0,1,2$,

$$
H_{h r}^{i}(M)=H_{D R}^{i}(M)=H^{i}(\mathfrak{g})=H_{h r}^{i}(\mathfrak{g}) .
$$

Moreover,

$$
\begin{aligned}
P^{m-k}(M) & =\left\{v \in H_{D R}^{m-k}(M) \mid L^{k+1} v=0\right\} \\
& =\left\{w \in H^{m-k}(\mathfrak{g}) \mid L^{k+1} w=0\right\} \\
& =P^{m-k}(\mathfrak{g}) .
\end{aligned}
$$

By Lemma 4.3 and the assumption of induction, we have

$$
H_{h r}^{m-k}(M)=P^{m-k}(M)+L\left(H_{h r}^{m-k-2}(M)\right)
$$




$$
\begin{aligned}
& =P^{m-k}(\mathfrak{g})+L\left(H_{h r}^{m-k-2}(\mathfrak{g})\right) \\
& =H_{h r}^{m-k}(\mathfrak{g}) .
\end{aligned}
$$

As in Proposition 4.4 , by the above argument and $L^{k}: \mathcal{H}^{m-k}(\mathfrak{g}) \rightarrow \mathcal{H}^{m+k}(\mathfrak{g})$ is an isomorphism, we have

$$
H_{h r}^{q}(M)=H_{h r}^{q}(\mathfrak{g}) \quad(q=0, \ldots, 2 m),
$$

where $\left(M^{2 m}, \omega\right)$ is a symplectic nilmanifold such that $\omega$ is a left $G$-invariant closed 2-form.

Let $(M, \omega)$ be a compact symplectic nilmanifold, then by Nomizu's theorem there exists $\omega_{0}$ which is a left $G$-invariant closed 2-form such that $\omega-\omega_{0}=d \gamma$. Moreover, $\omega_{0}$ is also non-degenerate (Since $\omega^{m}-\omega_{0}^{m}=d \tau$ for some $\tau \in \Omega^{2 m-1}(M)$ ). Therefore, by Proposition 4.4 and 4.5 ,

$$
H_{\omega-h r}^{q}(M)=H_{\omega_{0}-h r}^{q}(M)=H_{\omega_{0}-h r}^{q}(\mathfrak{g}) .
$$

Then we assume that symplectic structures on $M=G / \Gamma$ are left $G$-invariant to study harmonic cohomology groups on a compact nilmanifold $M$.

From now on we always assume that $(M, \omega)$ is a compact symplectic $(r+1)$-step nilmanifold. Let $\mathfrak{g}$ be an $(r+1)$-step nilpotent Lie algebra. Consider the descending central series $\left\{\mathfrak{g}^{(i)}\right\}$ of $\mathfrak{g}$, where $\mathfrak{g}^{(i+1)}=\left[\mathfrak{g}, \mathfrak{g}^{(i)}\right]$ and $\mathfrak{g}^{(0)}=\mathfrak{g}$. Let $\mathfrak{a}^{(i)}$ denote a vector space complement of $\mathfrak{g}^{(i+1)}$ in $\mathfrak{g}^{(i)}$ :

$$
\mathfrak{g}^{(i)}=\mathfrak{g}^{(i+1)}+\mathfrak{a}^{(i)}
$$

for $i=0,1, \ldots, r-1$ and define $n_{i}=\operatorname{dim} \mathfrak{a}^{(i)}$. For simplicity let $\bigwedge^{i_{0}} \mathfrak{a}^{(0)}{ }^{*} \wedge \cdots \wedge$ $\bigwedge^{i_{r}} \mathfrak{a}^{(r)^{*}}=\bigwedge^{i_{0}, \ldots, i_{r}}$. Then

$$
\bigwedge^{s} \mathfrak{g}^{*}=\sum_{i_{0}+\cdots+i_{r}=s} \bigwedge^{i_{0}, \ldots, i_{r}} .
$$

Lemma 4.6 (Benson-Gordon [1]).

$$
H^{1}(\mathfrak{g})=Z^{1}(\mathfrak{g})=\bigwedge^{1,0, \ldots, 0} .
$$

Lemma 4.7 (Benson-Gordon [1]). Any closed 2-form $\sigma \in \bigwedge^{2} \mathfrak{g}^{*}$ belongs to $\bigwedge^{1,0, \ldots, 0,1}+\sum \bigwedge^{i_{0}, \ldots, i_{r-1}, 0}$.

Let $\lambda_{1}, \ldots, \lambda_{n_{r}}$ be a basis of $\bigwedge^{0, \ldots, 0,1}$. By Lemma 4.9 , the invariant symplectic form $\omega$ can be written as

$$
\omega=\beta_{1} \wedge \lambda_{1}+\cdots+\beta_{n_{r}} \wedge \lambda_{n_{r}} \text { modulo } \sum \bigwedge^{i_{0}, \ldots, i_{r-1}, 0},
$$


where $\beta_{1}, \ldots, \beta_{n_{r}}$ are elements of $\bigwedge^{1,0, \ldots, 0}$. By non-degeneracy of $\omega, \beta_{1}, \ldots, \beta_{n_{r}}$ are linearly independent and thus can be extended to a basis

$$
\beta_{1}, \ldots, \beta_{n_{r}}, \ldots, \beta_{n_{0}}
$$

for $\bigwedge^{1,0, \ldots, 0}$.

Lemma 4.8 (Benson-Gordon [1]). (1) $\bigwedge^{2 m-1}\left(\mathfrak{g}^{*}\right)=Z^{2 m-1}(\mathfrak{g})$.

(2) $\sigma \in B^{2 m-1}(\mathfrak{g})$ is divisible by $\beta_{1} \wedge \cdots \wedge \beta_{n_{0}}$.

Hence by this lemma, $B^{2 m-1}(\mathfrak{g}) \subset \sum_{n_{0}+i_{1}+\cdots+i_{r}=2 m-1} \bigwedge^{n_{0}, i_{1}, \ldots, i_{r}}$. However, since $\operatorname{dim} B^{2 m-1}(\mathfrak{g})=\operatorname{dim} Z^{2 m-1}(\mathfrak{g})-\operatorname{dim} H^{2 m-1}(\mathfrak{g})=\operatorname{dim} Z^{2 m-1}(\mathfrak{g})-\operatorname{dim} H^{1}(\mathfrak{g})=n_{1}+$ $\cdots+n_{r}=\operatorname{dim} \sum \bigwedge^{n_{0}, i_{1}, \ldots, i_{r}}$, we have $B^{2 m-1}(\mathfrak{g})=\sum_{n_{0}+i_{1}+\cdots+i_{r}=2 m-1} \bigwedge^{n_{0}, i_{1}, \ldots, i_{r}}$.

\section{Proof of Theorem 1}

In this section, we prove Theorem 1 and some propositions. We use same notations introduced in Section 4.

Let $(G / \Gamma, \omega)$ be a compact symplectic $(r+1)$-step nilmanifold. By Nomizu's theorem, there exists a left $G$-invariant closed 2-form such that $\omega-\omega_{0}=d \gamma$ for some $\gamma \in \Omega^{1}(G / \Gamma)$. (Moreover, $\omega_{0}$ is non-degenerate). Therefore, by Proposition 4.4, we only consider the case that a symplectic form is a left $G$-invariant closed form.

Proof of Theorem 1. Note that $L^{m-1}: \mathcal{H}^{1}(\mathfrak{g}) \rightarrow \mathcal{H}^{2 m-1}(\mathfrak{g})$ is an isomorphism and $Z^{1}(\mathfrak{g})=\mathcal{H}^{1}(\mathfrak{g})$. For $i=1, \ldots, n_{r}$, consider $\beta_{i} \in Z^{1}(\mathfrak{g})=H^{1}(\mathfrak{g})$. Since $\omega^{m-1} \in$ $\sum_{i_{0}+\cdots+i_{r}=2 m-2} \bigwedge^{i_{0}, \ldots, i_{r}}$ is a $(2 m-2)$-form, we see $i_{0}=n_{0}, n_{0}-1, n_{0}-2$. Then $\omega^{m-1}$ can be written as $\omega^{m-1}=\delta_{1}+\delta_{2}^{\prime}+\delta_{2}^{\prime \prime}$, where $\delta_{1}, \delta_{2}^{\prime}, \delta_{2}^{\prime \prime}$ are $(2 m-2)$-forms such that $\delta_{1} \in \bigwedge^{n_{0}-2, n_{1}, \ldots, n_{r}}, \delta_{2}^{\prime} \in \sum \bigwedge^{n_{0}-1, i_{1}, \ldots, i_{r}}$ and $\delta_{2}^{\prime \prime} \in \sum \bigwedge^{n_{0}, i_{1}, \ldots, i_{r}}$. Hence

$$
L^{m-1} \beta_{i}=\beta_{i} \wedge \delta_{1}+\beta_{i} \wedge \delta_{2}^{\prime}+\beta_{i} \wedge \delta_{2}^{\prime \prime} .
$$

We claim that $L^{m-1} \beta_{i}$ is an exact form. Since each term of $\delta_{1}$ is divisible by $\lambda_{1} \wedge \cdots \wedge$ $\lambda_{n_{r}}$ and hence also by $\beta_{1} \wedge \cdots \wedge \beta_{n_{r}}$. Thus we get $\beta_{i} \wedge \delta_{1}=0$ (Note that $i=1, \ldots, n_{r}$ ). Moreover, by Lemma 4.8, we get

$$
\beta_{i} \wedge \delta_{2}^{\prime} \in \sum \bigwedge^{n_{0}, i_{1}, \ldots, i_{r}}=B^{2 m-1}(\mathfrak{g}) .
$$

Hence we now have

$$
L^{m-1} \beta_{i}=\beta_{i} \wedge \omega^{m-1} \in B^{2 m-1}(\mathfrak{g}) .
$$

It follows that

$$
\operatorname{dim} H_{h r}^{1}(M)-\operatorname{dim} H_{h r}^{2 m-1}(M) \geq \operatorname{dim} \mathfrak{g}^{(r)} .
$$


Furthermore,

$$
\begin{aligned}
\operatorname{dim} & H_{h r}^{1}(\mathfrak{g})-\operatorname{dim} H_{h r}^{2 m-1}(\mathfrak{g}) \\
= & \operatorname{dim} \mathcal{H}^{1}(\mathfrak{g})-\operatorname{dim}\left(B^{1}(\mathfrak{g}) \cap \mathcal{H}^{1}(\mathfrak{g})\right) \\
& \quad-\operatorname{dim} \mathcal{H}^{2 m-1}(\mathfrak{g})+\operatorname{dim}\left(B^{2 m-1}(\mathfrak{g}) \cap \mathcal{H}^{2 m-1}(\mathfrak{g})\right) \\
= & \operatorname{dim}\left(B^{2 m-1}(\mathfrak{g}) \cap \mathcal{H}^{2 m-1}(\mathfrak{g})\right)-\operatorname{dim}\left(B^{1}(\mathfrak{g}) \cap \mathcal{H}^{1}(\mathfrak{g})\right) \\
= & \operatorname{dim}\left(B^{2 m-1}(\mathfrak{g}) \cap \mathcal{H}^{2 m-1}(\mathfrak{g})\right) \\
\leq & \operatorname{dim}\left(B^{2 m-1}(\mathfrak{g})\right)=n_{1}+\cdots+n_{r} .
\end{aligned}
$$

Thus, in particular $M$ is a 2-step nilmanifold, we have

$$
\operatorname{dim} H_{h r}^{1}(M)-\operatorname{dim} H_{h r}^{2 m-1}(M)=n_{1} .
$$

Proposition 5.1. Let $\left(M^{2 m}=G / \Gamma, \omega\right)$ be a compact symplectic $(r+1)$-step nilmanifold. Assume that $\operatorname{dim} \mathfrak{g}-\operatorname{dim}[\mathfrak{g}, \mathfrak{g}]-2<\operatorname{dim} \mathfrak{g}^{(r)}$. Then we have

$$
\operatorname{dim} H_{h r}^{1}(M)-\operatorname{dim} H_{h r}^{2 m-1}(M)=n_{0}=\operatorname{dim} \mathfrak{g}-\operatorname{dim}[\mathfrak{g}, \mathfrak{g}] .
$$

Proof. Let

$$
\left\{\beta_{1}, \ldots, \beta_{n_{r}}, \ldots, \beta_{n_{0}}\right\}
$$

be a basis of $\bigwedge^{1,0, \ldots, 0}$. As in proof of Theorem 1 , we write $\omega^{m-1}$ as $\omega^{m-1}=\delta_{1}+\delta_{2}^{\prime}+\delta_{2}^{\prime \prime}$, $\delta_{1} \in \bigwedge^{n_{0}-2, n_{1}, \ldots, n_{r}}, \delta_{2}^{\prime} \in \sum \bigwedge^{n_{0}-1, i_{1}, \ldots, i_{r}}$ and $\delta_{2}^{\prime \prime} \in \sum \bigwedge^{n_{0}, i_{1}, \ldots, i_{r}}$. By our assumption, we see that $\delta_{1}=0$. Then, for any $i=1, \ldots, n_{0}, \beta_{i} \wedge \omega^{m-1} \in B^{2 m-1}(\mathfrak{g})$.

Proposition 5.2. Let $\left(M^{2 m}=G / \Gamma, \omega\right)$ be a compact symplectic 3-step nilmanifold such that $n_{1}=1$. Then

$$
\operatorname{dim} H_{h r}^{1}(M)-\operatorname{dim} H_{h r}^{2 m-1}(M)=n_{1}+n_{2} .
$$

Proof. We may assume that the symplectic form $\omega$ can be written as

$$
\omega=\beta_{1} \wedge \lambda_{1}+\cdots+\beta_{n_{2}} \wedge \lambda_{n_{2}}+\beta_{n_{2}+n_{1}} \wedge \tau \text { modulo } \bigwedge^{2,0,0},
$$

where $\tau \in \mathfrak{a}^{(1)^{*}}$. Then we see $\delta_{1} \in \bigwedge^{n_{0}-2, n_{1}, n_{2}}$ is divisible by $\beta_{n_{2}+n_{1}}$.

Remark. It is not true that, if $\left(M^{2 m}=G / \Gamma, \omega\right)$ be an $(r+1)$-step compact symplectic nilmanifold such that $n_{1}=n_{2}=\cdots=1$, then $\operatorname{dim} H_{h r}^{1}(M)-\operatorname{dim} H_{h r}^{2 m-1}(M)=$ $n_{1}+n_{2}+\cdots+n_{r}$. For example, consider the Lie algebra $\mathfrak{g}=\mathfrak{n}_{1}^{2 m-1} \times \mathfrak{a}$ where $\mathfrak{a}$ is a Lie algebra for dimension 1 and

$$
\mathfrak{n}_{1}^{2 m-1}=\operatorname{span}\left\{X_{1}, \ldots, X_{2 m-1}\right\}
$$


where $\left[X_{1}, X_{i}\right]=X_{i-1}(i=3, \ldots, 2 m-1)$. Let $\left\{\omega_{1}, \ldots, \omega_{2 m-1}\right\}$ (resp. $\left.\omega_{2 m}\right)$ be the dual basis of $\mathfrak{n}_{1}^{2 m-1}$ (resp. $\left.\mathfrak{a}\right)$ and

$$
\omega=\omega_{1} \wedge \omega_{2 m}+\sum_{i=0}^{m-2}(-1)^{i} \omega_{2+i} \wedge \omega_{2 m-1-i},
$$

then

$$
\operatorname{dim} H_{h r}^{1}(\mathfrak{g})-\operatorname{dim} H_{h r}^{2 m-1}(\mathfrak{g})=1 .
$$

Moreover, we can easily see that for any symplectic form on $M, \operatorname{dim} H_{h r}^{1}(M)-$ $\operatorname{dim} H_{h r}^{2 m-1}(M)=1$.

Proposition 5.3. Let $\left(M^{2 m}, \omega\right)$ be a compact symplectic $(r+1)$-step nilmanifold such that $n_{0}-2=n_{r}$. Let $\omega^{m-1}=\delta_{1}+\delta_{2}^{\prime}+\delta_{2}^{\prime \prime}$, where $\delta_{1}, \delta_{2}^{\prime}, \delta_{2}^{\prime \prime}$ are $(2 m-2)$-forms such that $\delta_{1} \in \bigwedge^{n_{0}-2, n_{1}, \ldots, n_{r}}, \delta_{2}^{\prime} \in \sum \bigwedge^{n_{0}-1, i_{1}, \ldots, i_{r}}, \delta_{2}^{\prime \prime} \in \sum \bigwedge^{n_{0}, i_{1}, \ldots, i_{r}}$. Then we have

$$
\begin{aligned}
& \delta_{1} \neq 0 \Rightarrow \operatorname{dim} H_{h r}^{1}(M)-\operatorname{dim} H_{h r}^{2 m-1}(M)=n_{r} \\
& \delta_{1}=0 \Rightarrow \operatorname{dim} H_{h r}^{1}(M)-\operatorname{dim} H_{h r}^{2 m-1}(M)=n_{0} .
\end{aligned}
$$

Proof. We may assume that the symplectic form $\omega$ can be written as

$$
\omega=\beta_{1} \wedge \lambda_{1}+\cdots+\beta_{n_{r}} \wedge \lambda_{n_{r}} \text { modulo } \sum \bigwedge^{i_{0}, \ldots, i_{r-1}, 0}
$$

Moreover, let $\left\{\beta_{1}, \ldots, \beta_{n_{r}}, \beta_{n_{r}+1}, \beta_{n_{r}+2}\right\}$ be a basis of $\left(\mathfrak{a}^{(0)}\right)^{*}$. By our assumption, we see

$$
\omega^{m-1}=a \beta_{1} \wedge \cdots \wedge \beta_{n_{r}} \wedge \lambda_{1} \wedge \cdots \wedge \lambda_{n_{r}} \wedge \tau+\delta_{2}^{\prime}+\delta_{2}^{\prime \prime},
$$

where $a \neq 0$ and $\tau \in \bigwedge^{0, i_{1}, \ldots, i_{n_{r}}}$. Hence,

$$
\begin{aligned}
& \beta_{n_{r}+1} \wedge \omega^{m-1} \neq 0 \\
& \beta_{n_{r}+2} \wedge \omega^{m-1} \neq 0 .
\end{aligned}
$$

Assume that $\beta_{n_{r}+1} \wedge \omega^{m-1}$ and $\beta_{n_{r}+2} \wedge \omega^{m-1}$ belong to same cohomology class of $H^{2 m-1}(\mathfrak{g})$. Therefore

$$
\begin{gathered}
\beta_{n_{r}+1} \wedge \omega^{m-1}-\beta_{n_{r}+2} \wedge \omega^{m-1}=d \gamma \\
0 \neq \beta_{n_{r}+1} \wedge \delta_{1}-\beta_{n_{r}+2} \wedge \delta_{1}=-\beta_{n_{r}+1} \wedge \delta_{2}^{\prime}+\beta_{n_{r}+2} \wedge \delta_{2}^{\prime}-\beta_{n_{r}+1} \wedge \delta_{2}^{\prime \prime}+\beta_{n_{r}+2} \wedge \delta_{2}^{\prime \prime}+d \gamma
\end{gathered}
$$

The right hand side is divisible by $\beta_{1} \wedge \cdots \wedge \beta_{n_{r}+2}$. Conversely, the left hand side is not divisible by $\beta_{1} \wedge \cdots \wedge \beta_{n_{r}+2}$. It is a contradiction.

(2) It is obvious. 


\section{Examples}

Now we shall give some examples of compact symplectic nilmanifold $G / \Gamma$. Since each Lie algebra $\mathfrak{g}$ has a basis with respect to which the constants of structure are rational, the simply-connected Lie group $G$ corresponding to $\mathfrak{g}$ admits a lattice.

EXAMPLE 6.1 (A generalization of Heisenberg group) ([3]). Let us consider the following Lie algebra.

$$
\mathfrak{h}(1, p)=\operatorname{span}\left\{X_{1}, \ldots, X_{p}, Y, Z_{1}, \ldots, Z_{p}\right\}
$$

where

$$
\left[X_{i}, Y\right]=Z_{i}
$$

and $\left\{\mu_{1}, \ldots, \mu_{p}, \nu, \lambda_{1}, \ldots, \lambda_{p}\right\}$ be its dual basis. Then we have

$$
d \mu_{i}=d \nu=0, \quad d \lambda_{i}=-\mu_{i} \wedge \nu .
$$

Thus $\mu_{i} \wedge \lambda_{i}$ is a closed 2-form.

Similarly, we also define

$$
\mathfrak{h}(1, q)=\operatorname{span}\left\{X_{1}^{\prime}, \ldots, X_{q}^{\prime}, Y^{\prime}, Z_{1}^{\prime} \ldots, Z_{q}^{\prime}\right\}
$$

and $\operatorname{span}\left\{\mu_{1}^{\prime}, \ldots, \mu_{q}^{\prime}, \nu^{\prime}, \lambda_{1}^{\prime}, \ldots, \lambda_{q}^{\prime}\right\}$. Then there exists a non-degenerate closed 2-form $\omega=\sum \mu_{i} \wedge \lambda_{i}+\sum \mu_{i}^{\prime} \wedge \lambda_{i}^{\prime}+\nu \wedge \nu^{\prime}$ on $\mathfrak{g}_{p, q}=\mathfrak{h}(1, p) \times \mathfrak{h}(1, q)$, Let $G_{p, q}$ be the simplyconnected Lie group corresponding to $\mathfrak{g}_{p, q}=\mathfrak{h}(1, p) \times \mathfrak{h}(1, q)$ and $M$ be a compact nilmanifold of $G_{p, q}$. Since $\mathfrak{g}_{p, q}$ is 2-step, we have

$$
\operatorname{dim} H_{h r}^{1}(M)-\operatorname{dim} H_{h r}^{2 p+2 q+1}(M)=p+q .
$$

REMARK. In particular, consider a symplectic form

$$
\omega=\sum \mu_{i} \wedge \lambda_{i}+\sum \mu_{i}^{\prime} \wedge \lambda_{i}^{\prime}+\nu \wedge \nu^{\prime}
$$

Then we get

$$
\operatorname{dim} H_{h r}^{2}\left(\mathfrak{g}_{p, q}\right)-\operatorname{dim} H_{h r}^{2 p+2 q}\left(\mathfrak{g}_{p, q}\right)={ }_{p+q} C_{2} .
$$

Proof. By a straightforward calculation, we have

$$
\begin{aligned}
H_{h r}^{2}\left(\mathfrak{g}_{p, q}\right)=\operatorname{span}\left\{\left[\mu_{i} \wedge \mu_{j}\right],\left[\mu_{i} \wedge \lambda_{i}\right],\left[\lambda_{i} \wedge \nu\right],\left[\mu_{i} \wedge \lambda_{j}+\mu_{j} \wedge \lambda_{i}\right],\right. \\
\\
{\left[\mu_{h}^{\prime} \wedge \mu_{k}^{\prime}\right],\left[\mu_{h}^{\prime} \wedge \lambda_{h}^{\prime}\right],\left[\lambda_{h}^{\prime} \wedge \nu^{\prime}\right],\left[\mu_{h}^{\prime} \wedge \lambda_{k}^{\prime}+\mu_{k}^{\prime} \wedge \lambda_{h}^{\prime}\right], } \\
{\left.\left[\mu_{i} \wedge \mu_{h}^{\prime}\right],\left[\mu_{i} \wedge \nu^{\prime}\right],\left[\nu \wedge \mu_{h}^{\prime}\right],\left[\nu \wedge \nu^{\prime}\right]\right\} . }
\end{aligned}
$$


Thus we consider the images of this basis by $\wedge \omega^{p+q-1}$. Now we define decomposable $(2(p+q)-2)$-forms as follows:

$$
\begin{aligned}
& \delta_{1}^{i j}=\alpha_{i j} \wedge \nu \wedge \beta \wedge \nu^{\prime}, \quad \delta_{2}^{s t}=\alpha \wedge \nu \wedge \beta_{s t} \wedge \nu^{\prime}, \quad \delta_{3}^{i s}=\alpha_{i} \wedge \nu \wedge \beta_{s} \wedge \nu^{\prime}, \\
& \delta_{4}^{i}=\alpha_{i} \wedge \beta, \quad \delta_{5}^{s}=\alpha \wedge \beta_{s},
\end{aligned}
$$

for $1 \leq i<j \leq p, 1 \leq s<t \leq p$, where

$$
\begin{aligned}
\alpha & =\mu_{1} \wedge \cdots \wedge \mu_{p} \wedge \lambda_{1} \wedge \cdots \wedge \lambda_{p} \\
\alpha_{i} & =\mu_{1} \wedge \cdots \wedge \hat{\mu}_{i} \wedge \cdots \wedge \mu_{p} \wedge \lambda_{1} \wedge \cdots \wedge \hat{\lambda}_{i} \wedge \cdots \wedge \lambda_{p} \\
\alpha_{i j} & =6 \mu_{1} \wedge \cdots \wedge \hat{\mu}_{i} \wedge \cdots \wedge \hat{\mu}_{j} \wedge \cdots \wedge \mu_{p} \wedge \lambda_{1} \wedge \cdots \wedge \hat{\lambda}_{i} \wedge \cdots \wedge \hat{\lambda}_{j} \wedge \cdots \wedge \lambda_{p}, \\
\beta & =\mu_{1}^{\prime} \wedge \cdots \wedge \mu_{q}^{\prime} \wedge \lambda_{1}^{\prime} \wedge \cdots \wedge \lambda_{q}^{\prime} \\
\beta_{s} & =\mu_{1}^{\prime} \wedge \cdots \wedge \hat{\mu}_{s}^{\prime} \wedge \cdots \wedge \mu_{q}^{\prime} \wedge \lambda_{1}^{\prime} \wedge \cdots \wedge \hat{\lambda}_{s}^{\prime} \wedge \cdots \wedge \lambda_{q}^{\prime} \\
\beta_{s t} & =\mu_{1}^{\prime} \wedge \cdots \wedge \hat{\mu}_{s}^{\prime} \wedge \cdots \wedge \hat{\mu}_{t}^{\prime} \wedge \cdots \wedge \mu_{q}^{\prime} \wedge \lambda_{1}^{\prime} \wedge \cdots \wedge \hat{\lambda}_{s}^{\prime} \wedge \cdots \wedge \hat{\lambda}_{t}^{\prime} \wedge \cdots \wedge \lambda_{q}^{\prime} .
\end{aligned}
$$

Furthermore we write

$$
\omega^{p+q-1}=\delta_{1}+\delta_{2}+\delta_{3}+\delta_{4}+\delta_{5}
$$

where

$$
\begin{aligned}
& \delta_{h}=\sum_{i<j} c_{h}^{i j} \delta_{h}^{i j}, \quad c_{h}^{i j} \in \mathbb{R} \quad(h=1,2,3), \\
& \delta_{k}=\sum_{i} c_{k}^{i} \delta_{k}^{i}, \quad c_{k}^{i} \in \mathbb{R} \quad(k=4,5) .
\end{aligned}
$$

With the notation, first consider the image for $\mu_{m} \wedge \mu_{n}$ by $\wedge \omega^{p+q-1}$, then

$$
\mu_{m} \wedge \mu_{n} \stackrel{\wedge \omega^{p+q-1}}{\longrightarrow} \mu_{m} \wedge \mu_{n} \wedge \delta_{1} .
$$

However, it is the image by $d$ for the scalar multiple of

$$
\mu_{1} \wedge \cdots \wedge \hat{\mu}_{m} \wedge \cdots \wedge \mu_{p} \wedge \lambda_{1} \wedge \cdots \wedge \hat{\lambda}_{n} \wedge \cdots \wedge \lambda_{p} \wedge \hat{\nu} \wedge \mu_{1}^{\prime} \wedge \cdots \wedge \mu_{q} \wedge \lambda_{1}^{\prime} \wedge \cdots \wedge \lambda_{q}^{\prime} \wedge \nu^{\prime}
$$

which implies that $\mu_{m} \wedge \mu_{n} \wedge \omega^{p+q-1}$ is exact. Similarly, we can see $\left(\mu_{m}^{\prime} \wedge \mu_{n}^{\prime}\right) \wedge \omega^{p+q-1}$ and $\left(\mu_{m} \wedge \mu_{n}^{\prime}\right) \wedge \omega^{p+q-1}$ are exact. Then

$$
\operatorname{dim} H_{h r}^{2}\left(\mathfrak{g}_{p, q}\right)-\operatorname{dim} H_{h r}^{2 p+2 q}\left(\mathfrak{g}_{p, q}\right) \geq{ }_{p+q} C_{2} .
$$

Next, note that

$$
\bigwedge^{n_{0}-3, n_{1}} \stackrel{d}{\longrightarrow} \bigwedge^{n_{0}-1, n_{1}-1}
$$




$$
\begin{array}{lll}
\bigwedge^{n_{0}-2, n_{1}-1} \stackrel{d}{\longrightarrow} \quad \bigwedge^{n_{0}, n_{1}-2}, \\
\bigwedge^{n_{0}-1, n_{1}-2} \stackrel{d}{\longrightarrow} \quad 0 \\
\bigwedge^{n_{0}, n_{1}-3} \stackrel{d}{\longrightarrow} \quad 0 .
\end{array}
$$

Therefore, since

$$
\lambda_{m} \wedge \nu \stackrel{\wedge \omega^{p+q-1}}{\longrightarrow} \lambda_{m} \wedge \nu \wedge \delta_{4} \in \bigwedge^{n_{0}-2, n_{1}}
$$

$\lambda_{m} \wedge \nu \wedge \omega^{p+q-1}$ is not exact. Similarly, $\lambda_{m}^{\prime} \wedge \nu^{\prime} \wedge \omega^{p+q-1}$ is not exact and by nondegeneracy of $\omega, \mu_{m} \wedge \lambda_{m} \wedge \omega^{p+q-1}$ and $\mu_{n}^{\prime} \wedge \lambda_{n}^{\prime} \wedge \omega^{p+q-1}$ are not exact.

Finally, consider the image for $\mu_{m} \wedge \lambda_{n}+\mu_{n} \wedge \lambda_{m}$ by $\wedge \omega^{p+q-1}$,

$$
\mu_{m} \wedge \lambda_{n}+\mu_{n} \wedge \lambda_{m} \stackrel{\wedge \omega^{p+q-1}}{\longrightarrow} \mu_{m} \wedge \lambda_{n} \wedge \delta_{1}+\mu_{n} \wedge \lambda_{m} \wedge \delta_{1}
$$

Indeed,

$$
\begin{aligned}
&\left(\mu_{m} \wedge \lambda_{n}+\wedge \mu_{n} \wedge \lambda_{m}\right) \wedge \delta_{1} \\
&=(-1)^{(p-2)+(p-m)+(p-n-1)}\left(\mu_{1} \wedge \cdots \wedge \hat{\mu}_{m} \wedge \cdots \wedge \mu_{p} \wedge \lambda_{1} \wedge \cdots \wedge \hat{\lambda}_{n} \wedge \cdots \wedge \lambda_{p} \wedge \nu\right. \\
&\left.\quad+\mu_{1} \wedge \cdots \wedge \hat{\mu}_{n} \wedge \cdots \wedge \mu_{p} \wedge \lambda_{1} \wedge \cdots \wedge \hat{\lambda}_{m} \wedge \cdots \wedge \lambda_{p} \wedge \nu\right) \wedge \Omega^{\prime},
\end{aligned}
$$

where $\Omega^{\prime}=\mu_{1}^{\prime} \wedge \cdots \wedge \mu_{q}^{\prime} \wedge \lambda_{1}^{\prime} \wedge \cdots \wedge \lambda_{q}^{\prime} \wedge \nu^{\prime}$.

However, the image by $d$ for $\mu_{1} \wedge \cdots \hat{\mu}_{m} \wedge \cdots \wedge \hat{\mu}_{n} \wedge \cdots \wedge \mu_{p} \wedge \lambda_{1} \wedge \cdots \wedge \lambda_{p} \wedge \hat{\nu} \wedge \Omega^{\prime}$ is $\pm\left\{\left(\mu_{1} \wedge \cdots \wedge \hat{\mu}_{n} \wedge \cdots \wedge \mu_{p} \wedge \lambda_{1} \wedge \cdots \wedge \hat{\lambda}_{m} \wedge \cdots \wedge \lambda_{p} \wedge \nu \wedge \Omega^{\prime}\right)-\left(\mu_{1} \wedge \cdots \hat{\mu}_{m} \wedge \cdots \wedge\right.\right.$ $\left.\left.\mu_{p} \wedge \lambda_{1} \wedge \cdots \hat{\lambda}_{n} \wedge \lambda_{p} \wedge \nu \wedge \Omega^{\prime}\right)\right\}$. Thus we now see that $\left(\mu_{m} \wedge \lambda_{n}+\mu_{n} \wedge \lambda_{m}\right) \wedge \omega^{p+q}$ is not exact. Similarly, $\left(\mu_{m}^{\prime} \wedge \lambda_{n}^{\prime}+\mu_{n}^{\prime} \wedge \lambda_{m}^{\prime}\right) \wedge \omega^{p+q}$ is not also exact.

EXAMPLE 6.2 ([5]). Let $\mathfrak{g}$ be the Lie algebra defined by

$$
\mathfrak{g}=\operatorname{span}\left\{X_{1}, \ldots, X_{2 m}\right\}
$$

where $\left[X_{1}, X_{i}\right]=X_{i-1}(i=3, \ldots, 2 m)$ and $\omega_{1}, \ldots, \omega_{2 m}$ be its dual basis. Thus we get

$$
\begin{aligned}
d \omega_{1} & =d \omega_{2 m}=0 \\
d \omega_{i-1} & =-\omega_{1} \wedge \omega_{i}(i=3, \ldots, 2 m) .
\end{aligned}
$$

Then $\omega_{1} \wedge \omega_{2}, \sum_{i=0}^{m-2}(-1)^{i} \omega_{3+i} \wedge \omega_{2 m-i}$ are closed 2-forms and $\mathfrak{g}$ has a non-degenerate closed 2-form,

$$
\omega=\omega_{1} \wedge \omega_{2}+\sum_{i=0}^{m-2}(-1)^{i} \omega_{3+i} \wedge \omega_{2 m-i} .
$$


Since $n_{0}-2=0<n_{r}$, we get by Proposition 5.1

$$
\operatorname{dim} H_{h r}^{1}(M)-\operatorname{dim} H_{h r}^{2 m-1}(M)=2,
$$

for a compact symplectic nilmanifold $M=G / \Gamma$.

\section{Proof of Theorem 3}

Proof of Theorem 3. Let $\alpha\left(\omega_{t}\right)=\operatorname{dim} H_{h r}^{2}(\mathfrak{g})-\operatorname{dim} H_{h r}^{4}(\mathfrak{g})$.

Since

$$
\left[X_{1}, X_{6}\right]=X_{5},\left[X_{1}, X_{4}\right]=X_{3},\left[X_{4}, X_{6}\right]=X_{2},
$$

we get $d \omega_{2}=-\omega_{4} \wedge \omega_{6}, d \omega_{3}=-\omega_{1} \wedge \omega_{4}, d \omega_{5}=-\omega_{1} \wedge \omega_{6}$. As in Section 6, the simply-connected Lie group $G$ corresponding to $\mathfrak{g}$ admits a lattice. By a straightforward calculation, we have

$$
\begin{aligned}
H^{2}(\mathfrak{g})=\operatorname{span}\{ & {\left[\omega_{1} \wedge \omega_{3}\right],\left[\omega_{1} \wedge \omega_{5}\right],\left[\omega_{2} \wedge \omega_{4}\right],\left[\omega_{2} \wedge \omega_{6}\right],\left[\omega_{3} \wedge \omega_{4}\right],\left[\omega_{5} \wedge \omega_{6}\right], } \\
& {\left.\left[\omega_{1} \wedge \omega_{2}+\omega_{3} \wedge \omega_{6}\right],\left[\omega_{3} \wedge \omega_{6}-\omega_{4} \wedge \omega_{5}\right]\right\} }
\end{aligned}
$$

and

$$
B^{4}(\mathfrak{g})=\operatorname{span}\left\{\omega_{1346}, \omega_{1456}, \omega_{1246}, \omega_{1236}+\omega_{1245}-\omega_{3456}\right\},
$$

where $\omega_{i j k h}$ means the element $\omega_{i} \wedge \omega_{j} \wedge \omega_{k} \wedge \omega_{h}$.

Moreover, let $B(t)=\left(b_{i j}\right)=\left(\omega_{t}\left(X_{i}, X_{j}\right)\right)$, then we can easily verify

$$
\operatorname{det}(B(t))=\left(1-3 t+3 t^{2}+t^{3}\right)^{2} .
$$

Therefore, let $x_{0}\left(x_{0} \fallingdotseq-3.8473\right)$ be the unique real solution of $1-3 t+3 t^{2}+t^{3}=0$, $\omega_{t}$ is non-degenerate for $t \neq x_{0}$

Next, we calculate the image of $L_{\omega_{t}}$ for $Z^{2}(\mathfrak{g})$. Note that $Z^{2}(\mathfrak{g})=\mathcal{H}^{2}(\mathfrak{g})$ and $L_{\omega_{t}}\left(\mathcal{H}^{2}(\mathfrak{g})\right)=\mathcal{H}^{4}(\mathfrak{g})$.

$$
\begin{aligned}
\omega_{1} & \wedge \omega_{3} \stackrel{\wedge \omega_{t}}{\longrightarrow}(1-t)\left(\omega_{1324}+\omega_{1356}\right)-t \omega_{1345} \\
\omega_{1} & \wedge \omega_{5} \stackrel{\wedge \omega_{t}}{\longrightarrow}(1-t) \omega_{1524}-t \omega_{1536} \\
\omega_{2} & \wedge \omega_{4} \stackrel{\wedge \omega_{t}}{\longrightarrow}(1-t)\left(\omega_{2413}+\omega_{2456}\right)-t \omega_{2436} \\
\omega_{2} & \wedge \omega_{6} \stackrel{\wedge \omega_{t}}{\longrightarrow}(1-t) \omega_{2613}-t \omega_{2645} \\
\omega_{3} & \wedge \omega_{4} \stackrel{\wedge \omega_{t}}{\longrightarrow}(1-t) \omega_{3456}-2 t \omega_{3412} \\
\omega_{5} & \wedge \omega_{6} \stackrel{\wedge \omega_{t}}{\longrightarrow}(1-t)\left(\omega_{5613}+\omega_{5624}\right)-2 t \omega_{5612} \\
\omega_{1} \wedge \omega_{2}+\omega_{3} & \wedge \omega_{6} \stackrel{\wedge \omega_{t}}{\longrightarrow}(1-t)\left(\omega_{1256}+\omega_{3624}\right)-t\left(3 \omega_{1236}+\omega_{1245}+\omega_{3645}\right) \\
\omega_{3} \wedge \omega_{6}-\omega_{4} & \wedge \omega_{5} \stackrel{\wedge \omega_{t}}{\longrightarrow}(1-t)\left(\omega_{3624}-\omega_{4513}\right)-t\left(2 \omega_{3612}-2 \omega_{4512}\right)
\end{aligned}
$$


Since $L_{\omega_{t}}\left(H^{2}(\mathfrak{g})\right) \subset \bigwedge^{2,2}$ and $\omega_{1346}, \omega_{1456}, \omega_{1246} \in \bigwedge^{3,1}$, we need only consider the dimension of $\operatorname{span}\left\{L_{\omega_{t}}\left(H^{2}(\mathfrak{g})\right), \omega_{1236}+\omega_{1245}-\omega_{3456}\right\}$, where $\omega_{1236}+\omega_{1245}-\omega_{3456}$ is a exact form.

Thus, we consider the matrix form of $\left\{L_{\omega_{t}}\left(\omega_{13}\right), L_{\omega_{t}}\left(\omega_{15}\right), L_{\omega_{t}}\left(\omega_{24}\right), L_{\omega_{t}}\left(\omega_{26}\right)\right.$, $\left.L_{\omega_{t}}\left(\omega_{34}\right), L_{\omega_{t}}\left(\omega_{56}\right), L_{\omega_{t}}\left(\omega_{12}-\omega_{36}\right), L_{\omega_{t}}\left(\omega_{36}-\omega_{45}\right), \omega_{1236}+\omega_{1245}-\omega_{3456}\right\}$ with respect to the basis $\left\{\omega_{1234}, \omega_{1236}, \omega_{1245}, \omega_{1256}, \omega_{1345}, \omega_{1356}, \omega_{2346}, \omega_{2456}, \omega_{3456}\right\}$ of $\bigwedge^{2,2}$, let $A(t)$ denote its matrix. Then we get the following matrix:

$$
A(t)=\left(\begin{array}{ccccccccc}
t-1 & 0 & 0 & 0 & -t & 1-t & 0 & 0 & 0 \\
0 & 0 & 1-t & 0 & 0 & t & 0 & 0 & 0 \\
t-1 & 0 & 0 & 0 & 0 & 0 & t & 1-t & 0 \\
0 & -1+t & 0 & 0 & 0 & 0 & 0 & -t & 0 \\
-2 t & 0 & 0 & 0 & 0 & 0 & 0 & 0 & 1-t \\
0 & 0 & 0 & -2 t & 0 & 1-t & 0 & 1-t & 0 \\
0 & -3 t & -t & 1-t & 0 & 0 & t-1 & 0 & -t \\
0 & -2 t & 2 t & 0 & t-1 & 0 & t-1 & 0 & 0 \\
0 & 1 & 1 & 0 & 0 & 0 & 0 & 0 & -1
\end{array}\right)
$$

Thus we have

$$
\operatorname{det} A(t)=12 t^{2}\left(1-3 t+3 t^{2}+t^{3}\right)^{2} .
$$

Hence, for $t \neq 0, x_{0}, \operatorname{det} A(t) \neq 0$. Then for $t \neq 0, x_{0}$,

$$
\alpha\left(\omega_{t}\right)=0 .
$$

On the other hand, since $\operatorname{det} A(t)=0$ for $t=0$,

$$
\alpha\left(\omega_{0}\right)=1 \text {. }
$$

In fact,

$$
\begin{aligned}
& \omega_{1} \wedge \omega_{3} \stackrel{\wedge \omega}{\longrightarrow} \omega_{1324}+\omega_{1356} \\
& \omega_{1} \wedge \omega_{5} \stackrel{\wedge \omega}{\longrightarrow} \omega_{1524} \\
& \omega_{2} \wedge \omega_{4} \stackrel{\wedge \omega}{\longrightarrow} \omega_{2413}+\omega_{2456} \\
& \omega_{2} \wedge \omega_{6} \stackrel{\wedge \omega}{\longrightarrow} \omega_{2613} \\
& \omega_{3} \wedge \omega_{4} \stackrel{\wedge \omega}{\longrightarrow} \omega_{3456} \\
& \omega_{5} \wedge \omega_{6} \stackrel{\wedge \omega}{\longrightarrow} \omega_{5613}+\omega_{5624} \\
& \omega_{1} \wedge \omega_{2}+\omega_{3} \wedge \omega_{6} \stackrel{\wedge \omega}{\longrightarrow} \omega_{1256}+\omega_{3624} \\
& \omega_{3} \wedge \omega_{6}-\omega_{4} \wedge \omega_{5} \stackrel{\wedge \omega}{\longrightarrow} \omega_{3624}+\omega_{4513}
\end{aligned}
$$

Thus, the image of $L_{\omega}$ for $\omega_{1} \wedge \omega_{5}-\omega_{2} \wedge \omega_{6}-\omega_{3} \wedge \omega_{4}$ is a exact form. Therefore, we now see

$$
\alpha\left(\omega_{0}\right)=\operatorname{dim} H_{h r}^{2}(\mathfrak{g})-\operatorname{dim} H_{h r}^{4}(\mathfrak{g})=1 .
$$




\section{References}

[1] C. Benson and C. Gordon: Kähler and Symplectic structures on nilmanifolds, Topology 27 (1988), 513-518.

[2] J.L. Brylinski: A differential complex for Poisson Manifolds, J. Differential Geometry 28 (1988), 93-114.

[3] L. Cordero, M. Fernandez and A. Gray: Symplectic manifolds with no Kähler structure, Topology 25 (1986), 375-380.

[4] M. Fernández, R. Ibáñez and M. de León: A Nomizu’s theorem for coeffective cohomology, Math. Z. 226 (1997), 11-23.

[5] M. Goze and Y. Khakimdjnov: Nilpotent Lie algebras, Kluwer academic publisher, 1996.

[6] O. Mathieu: Harmonic cohomology classes of symplectic manifolds, Comment. Math. Helv. 70 (1995), 1-9.

[7] Y. Matsushima: On the discrete subgroups and homogeneous spaces of nilpotent Lie groups, Nagoya Math. J. 2 (1951), 143-154.

[8] K. Nomizu: On the cohomology of compact homogeneous spaces of nilpotent Lie groups, Ann. of Math. 59 (1954), 531-538.

[9] M.S. Raghunathan: Discrete subgroups of Lie groups, Springer-Verlag, Berlin, 1972.

[10] A. Tralle and J. Oprea: Symplectic Manifolds with no Kähler Structure, SpringerVerlag, Berlin, 1997.

[11] D. Yan: Hodge structure on symplectic manifolds, Adv. in Math. 120 (1996), 143-154.

Department of Mathematics

Graduate School of Science

Osaka University

Toyonaka, Osaka 560-0043, Japan

e-mail: smv198yt@ecs.cmc.osaka-u.ac.jp 